(2) Open Access Full Text Article

\title{
Dynamics of Epicardiac Fat and Heart Function in Type 2 Diabetic Patients Initiated with SGLT-2 Inhibitors
}

This article was published in the following Dove Press journal: Diabetes, Metabolic Syndrome and Obesity: Targets and Therapy

\author{
Adina Braha (D) \\ Bogdan Timar (iD ${ }^{2}$ \\ Laura Diaconu ${ }^{3}$ \\ Raluca Lupusoru ${ }^{1,4}$ \\ Lucian Vasiluta ${ }^{3}$ \\ Alexandra Sima $\mathbb{1 D}^{3}$ \\ Adrian Vlad (iD ${ }^{3}$ \\ Mircea Munteanu ${ }^{3}$ \\ Alin Albai ${ }^{3}$ \\ Daniela Cipu (D) ${ }^{5}$ \\ Romulus Timar $^{3}$
}

'First Department of Internal Medicine, "Victor Babes" University of Medicine and Pharmacy, Timisoara, Romania; ${ }^{2}$ Department of Functional Sciences, "Victor Babes" University of Medicine and Pharmacy, Timisoara, Romania;

${ }^{3}$ Second Department of Internal

Medicine, "Victor Babes" University of

Medicine and Pharmacy, Timisoara,

Romania; ${ }^{4}$ Department of

Gastroenterology and Hepatology,

"Victor Babes" University of Medicine and Pharmacy, Timisoara, Romania;

${ }^{5}$ Department of Orthopedics-

Traumatology, Urology and Medical Imaging, "Victor Babes" University of Medicine and Pharmacy, Timisoara, Romania
Correspondence: Bogdan Timar Department of Functional Sciences, "Victor Babes" University of Medicine and Pharmacy, 2 Eftimie Murgu, Timisoara 30004I, Romania

Tel +40 74I 528093

$\mathrm{Fax}+40256462856$

Email timar.bogdan@umft.ro
Purpose: The aim of this study was to assess the dynamics of epicardiac adipose tissue (EAT) thickness and total volume as well as that of systolic and diastolic dysfunction in a group of patients with type 2 diabetes (T2D) after initiation of sodium glucose cotransporter 2 (SGLT 2) inhibitors therapy.

Patients and methods: This prospective, observational study included 53 patients with T2D who received SGLT-2 inhibitors for 24 weeks. In all patients, echocardiographic screening for EAT, systolic and diastolic dysfunction and non-contrast computed tomography scans were performed, both before and after 24 weeks of SGLT-2 inhibition. Imagistic evaluation was followed by the association's analysis between the dynamics of EAT and heart function, as well as the patient's clinical and biological parameters. We considered a decrease or increase of more than $10 \%$ in EAT as being clinically significant.

Results: The mean volume of EAT decreased significantly after SGLT 2 inhibition $(37.8 \pm 17.2$ vs. $\left.20.7 \pm 7 \mathrm{~cm}^{3} ; p<0.001\right)$. Median values of EAT thickness also decreased significantly (5.95 vs. $3.01 \mathrm{~mm}$; $<<0.001)$. Most patients, $75.4 \%$ (40/53), presented more than $10 \%$ decrease in EAT volume, $9.5 \%(5 / 53)$ had stable EAT volume values, while in $15.1 \%(8 / 53)$ the means of EAT volume increased. $73.5 \%$ of the patients had diastolic dysfunction type 1 (DD 1) at baseline. No significant change was observed in the left ventricular ejection fraction or diastolic dysfunction after 24 weeks of treatment. Although not statistically significant, an improvement in cardiac function has been noticed throughout the duration of 1 year of treatment with SGLT 2 inhibitors.

Conclusion: This study showed the beneficial effect of SGLT 2 inhibitors on EAT after a short period of treatment, but there were no significant changes in the systolic function during the 1st year of study. However, reducing epicardial fat has led to remission of diastolic dysfunction.

Keywords: epicardiac fat, diastolic dysfunction, heart failure

\section{Introduction}

In the past few years, epicardial fat has attracted a lot of interest as a marker of cardiovascular dysfunction and also as a therapeutic target. Epicardial fat has multiple roles within the heart: mechanical protection for coronary artery, ${ }^{1}$ supports the myocardium energy through a higher metabolism rate of free fatty acid, ${ }^{2}$ thermogenic functions, ${ }^{3}$ production of active biomarkers ${ }^{4-8}$ that can be harmful or beneficial to the heart morphology and function. Recent findings suggest that epicardial adipose tissue is associated with myocardium remodeling with induced fibrosis due to its 
secretion of pro-inflammatory cytokines ${ }^{9}$ and may lead to increased left ventricular mass and affects the diastolic heart function first. ${ }^{11,12}$

Along with heart failure, diabetes is a global health burden with a high prevalence in the general population that has been steadily increasing, reducing life quality and being associated with increased morbidity. Patients with diabetes have an increased risk of atherosclerotic cardiovascular events and heart failure, despite the control of cardiovascular risk factors and optimal HbAlc. ${ }^{13}$ The coexistence of the two entities, diabetes and heart failure, is quite common and is associated with a higher risk of hospitalization for heart failure and mortality of any cause or due to cardiovascular disease. ${ }^{14}$

Currently, choosing the right antidiabetic treatment is a challenge for practitioners. The latest treatment guidelines recommend that cardiovascular safety be at least ensured in the treatment of type 2 diabetes, although ideally, the cardiovascular risk should be reduced. Of all classes of antidiabetic agents, sodium glucose 2 cotransporter inhibitors have shown reduced risk of hospitalization for heart failure, as shown in a meta-analysis for 3 cardiovascular outcome trials - EMPAREG, CANVAS program and DECLARE - TIMI - 58. ${ }^{15}$

The aim of this study was to evaluate the dynamics of epicardiac fat and heart function after SGLT 2 inhibition in a group of type 2 diabetic patients and also to analyze the relation between epicardiac fat, type 1 diastolic dysfunction, obesity indices and metabolic outcomes, during a period of up to one year of treatment.

\section{Materials and Methods}

\section{Patients and Study Design}

A sample of 80 patients with T2D from Diabetes Clinic and Diabetes Center of "Pius Brinzeu" Emergency Hospital, Timisoara, were recruited in a prospective observational study over a 6-month period. Patients were recommended for this study by their prescribing physician before starting treatment with dapagliflozin. Eligible patients for this study met the following criteria: age over 18 years, type 2 diabetic, $\mathrm{HbA} 1 \mathrm{c}>7 \%$, glomerular filtration rate (GFR) $>60 \mathrm{ml} / \mathrm{min} /$ $1.73 \mathrm{~m}^{2}$, and had consented to being part of the study through a signed form handed in prior to any investigation. Exclusion criteria were: pregnancy during the study, lactation, type 1 diabetes of other types than type 2 diabetes, history of diabetes ketoacidosis, $\mathrm{HbAlc}<7 \%$, GFR $<60 \mathrm{ml} / \mathrm{min} / 1.73 \mathrm{~m}^{2}$, $\mathrm{BMI}<25 \mathrm{~kg} / \mathrm{m}^{2}$, history of repeated urinary infections or urogenital infections developed during the study or other side effects that required discontinuation of treatment with dapagliflozin, or the patient's refusal to take part in the study. We initially recruited 68 patients who were scheduled for follow-up visits after 3 and 6 months, respectively. At the 3 -month visits, a number of them were removed from the study and others needed intensification of diabetic treatment as recommended by the American Diabetes Association guidelines. Finally, 53 patients of 68 were clinically, biologically and imagisticaly reevaluated after 24 weeks of treatment with $10 \mathrm{mg}$ of dapagliflozin per day. At the 48week follow-up appointment, 45 patients who had been on SGLT 2 inhibitors were reevaluated from an echocardiographic point of view.

All patients signed a written consent form. The study was approved by the ethical committee of "Pius Brinzeu" Emergency County Hospital and was led in accordance with the Helsinki Declaration.

\section{Clinical and Anthropometric Assessments}

Age, medical history, chronic degenerative complications developed during the evolution of diabetes, the presence of hypertension and current treatment followed in the ambulatory were evaluated in each patient. All patients were measured for weight, height and waist circumference. The blood pressure was taken after the subject had been seated for at least five minutes, using a calibrated and certified Omron blood pressure monitor. We calculated body mass index as body weight divided by squared height, body surface area using Du Bois and Du Bois formula.

\section{Biochemical Assessments}

Blood and urine samples were collected before initiation of dapagliflozin and after 6 months of treatment, for routine lab parameters: glycaemia, total cholesterol, HDL cholesterol, LDL cholesterol, triglycerides, uric acid, $\mathrm{HbAlc}$, serum creatinin, urinary creatinin, urinary albumin, liver transaminases (AST, ALT), serum electrolytes (sodium, potassium). We used CKD-EPI formula to obtain GFR and we calculated urinary albumin/creatinin ratio (UACr).

\section{Imagistic Assessments}

We obtained information about epicardiac adipose tissue from all patients who underwent transthoracic, two-dimensionalguided M-mode cardiac ultrasound (Aloka SSD 4000). On non-contrast computed tomography (Philips MX 16), we measured different adipose tissue depots such as: epicardiac, subcutaneous, visceral fat at L4 vertebrae. To assess the 
reproducibility of cardiac ultrasound, the cardiologist performed a blind analysis of all recorded images of patients' heart function and epicardiac thickness twice. Epicardiac fat thickness was analyzed via two different methods: cardiac ultrasound (in the subcostal incidence, parasternal long axis, as the echo-free space between the liver and right ventricle wall) and non-contrast computed tomography (subcostal, anterior incidence). An example of the image analysis is presented in Figure 1. We extracted the images from native computed tomography and identified adipose tissue as any pixel with a threshold between -190 and -30 Hounsfield Units. ${ }^{10}$ All fat volumes were corrected to body surface area. We calculated subcutaneous/visceral fat volume ratio. Type 1 diastolic dysfunction was defined as $\mathrm{E} / \mathrm{A}<1$ and $\mathrm{E} / \mathrm{e}^{\text {' }}>15$. The CT scan was ECG gated to reduce the possible occurrence of artefacts.

\section{Statistical Analysis}

SPSS software, Version 20.0 (IBM SPSS Statistics) and Microsoft Office Excel 2019 were used for the statistical analysis. The distribution of numerical variables was tested with Kolmogorov-Smirnov test. We used $t$-test and ANOVA (for normal distribution) and MannWhitney $U$-test or Kruskal-Wallis tests (for non-normal distribution) to assess the differences between numerical variables. The proportions were analyzed using the Chisquare test. To compare the correlation between two diagnostic tests, we used the Bland Altman plot and Pearson correlation coefficient. Linear regression and logistic regression were used for the evaluation of the factors associated with type 1 diastolic dysfunction and epicardiac fat. A $p$ value $<0.05$ was considered significant for the statistical tests and $95 \%$ confidence intervals were calculated.

\section{Results}

\section{Patient Characteristics}

Of 53 patients who completed this study, 32 (60.3\%) were males. The average age of subjects was $57.6 \pm 10.3$ years. The evolution of diabetes in the study group ranged from 0 to 24 years, with an average of 7 years. Among these patients, 22 $(41.5 \%)$ had a history or treatment for myocardial infarction, angina or stroke, $22(41.5 \%)$ had symptoms of diabetic polyneuropathy, $4(7.5 \%)$ had peripheral arterial disease. The patients' characteristics are presented in Table 1. Table 2 shows the therapies for patients at baseline.

\section{Imagistic Examination}

Reproducibility of left ventricle ejection fraction measurements was tested to see if there are any variations and we

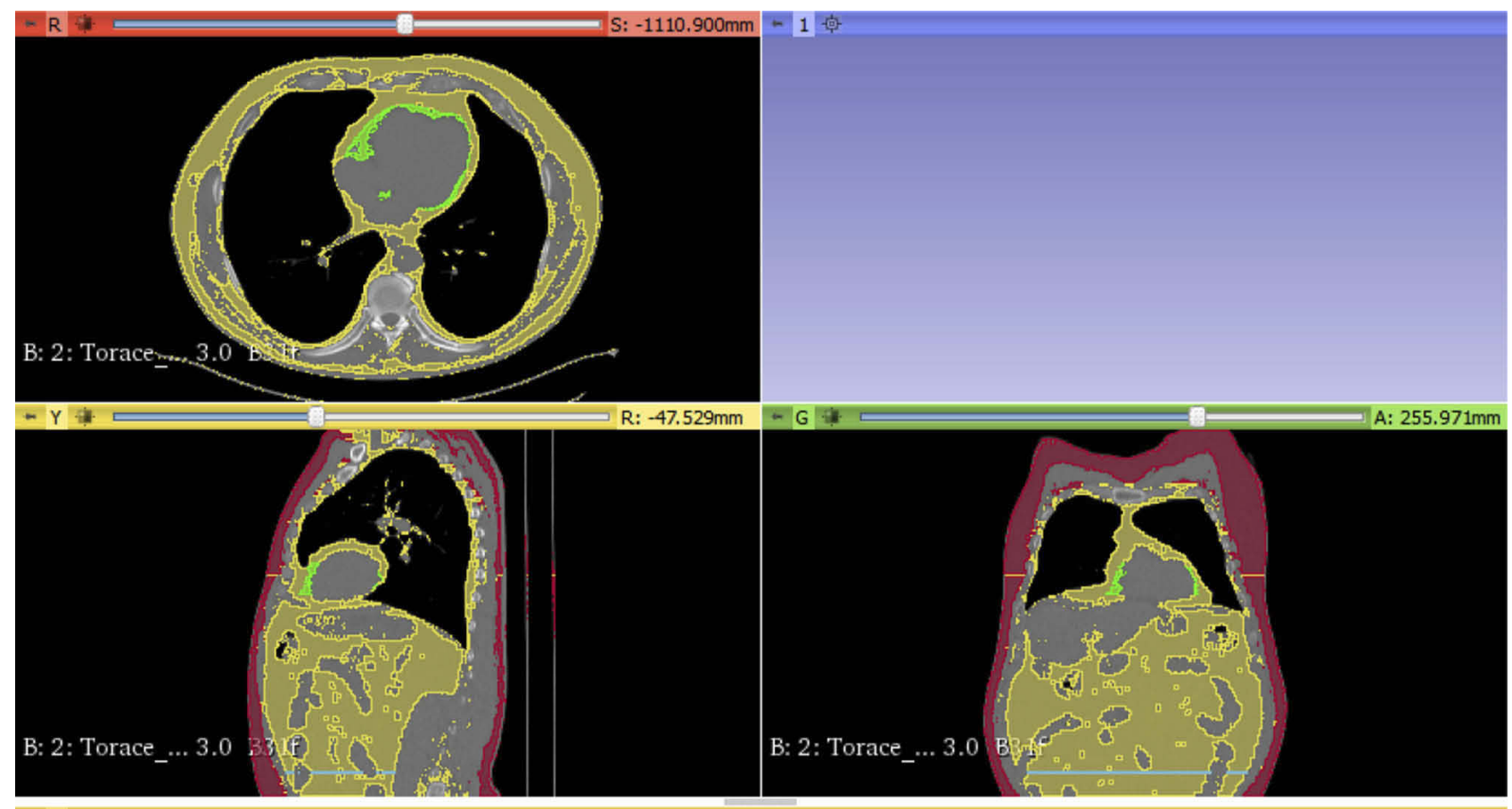

$-T$

Figure I Epicardiac fat volume (green), total visceral fat volume (yellow - second view), L4 vertebral visceral fat volume (blue line) and subcutaneous adipose tissue volume (red) measured with 3D Slicer software. 
Table I Patients' Characteristics

\begin{tabular}{|c|c|c|c|}
\hline Parameter & Baseline & At 24 Weeks Follow-Up, After Dapagliflozin & $p$-Value \\
\hline Weight $(\mathrm{kg})^{\mathrm{a}}$ & $99.1 \pm 15.9$ & $94.2 \pm 14.6$ & $<0.001$ \\
\hline BMI $\left(\mathrm{kg} / \mathrm{cm}^{2}\right)^{\mathrm{a}}$ & $34.5 \pm 4.7$ & $32.9 \pm 4.7$ & $<0.001$ \\
\hline Waist circumference $(\mathrm{cm})^{\mathrm{a}}$ & $115.2 \pm 11.4$ & $1|3.7 \pm| 1.3$ & 0.03 \\
\hline In women ${ }^{a}$ & $115.6 \pm 10.1$ & $115 \pm 11$ & 0.57 \\
\hline $\ln m^{a}{ }^{a}$ & $115 \pm 12.3$ & $112.9 \pm 11.7$ & 0.02 \\
\hline Total Cholesterol $(\mathrm{mg} / \mathrm{dl})^{\mathrm{a}}$ & $|92.5| \pm 6 \mid$ & $184.7 \pm 46.4$ & 0.23 \\
\hline Triglycerides $(\mathrm{mg} / \mathrm{dl})^{\mathrm{b}}$ & $17 \mid .5(55 ; 887)$ & | $46.5(58 ; 1397)$ & 0.28 \\
\hline $\mathrm{HDL}$ c $(\mathrm{mg} / \mathrm{dl})^{\mathrm{b}}$ & $38.5(17 ; 89)$ & $42(19 ; 90)$ & 0.01 \\
\hline In women ${ }^{a}$ & $39.5 \pm 12$ & $45.1 \pm 12.4$ & 0.0015 \\
\hline In men ${ }^{b}$ & $38(17 ; 89)$ & $42(19 ; 90)$ & 0.01 \\
\hline $\operatorname{LDL~c~(mg/dl~})^{\mathrm{a}}$ & $115.98 \pm 45.4$ & $103.4 \pm 38.3$ & 0.007 \\
\hline Uric acid $(\mathrm{mg} / \mathrm{dl})^{\mathrm{a}}$ & $5.16 \pm 1.27$ & $4.8 \pm 1.1$ & 0.007 \\
\hline Fasting glycaemia $(\mathrm{mg} / \mathrm{dl})^{\mathrm{a}}$ & $211.17 \pm 68.95$ & $157.6 \pm 42.8$ & $<0.001$ \\
\hline $\mathrm{HbAlc}(\%)^{\mathrm{a}}$ & $8.65 \pm 1.17$ & $7.8 \pm 1.2$ & 0.001 \\
\hline Sodium $(\mathrm{mmol} / \mathrm{l})^{\mathrm{a}}$ & $139.15 \pm 2.48$ & $\mid 39.1 \pm 2.4$ & 0.9 \\
\hline Potassium $(\mathrm{mmol} / \mathrm{l})^{\mathrm{b}}$ & $4.5(3.7 ; 5.8)$ & $4.5(3.7 ; 5.3)$ & 0.78 \\
\hline GFR $(\mathrm{mL} / \mathrm{min} / 173 \mathrm{~cm})^{\mathrm{a}}$ & $85.9 \pm 15.6$ & $93.3 \pm 14.2$ & $<0.001$ \\
\hline $\mathrm{UACr}(\mathrm{mg} / \mathrm{g})^{\mathrm{b}}$ & $15.8(5.5 ; 69 \mid .8)$ & $16.1(1.3 ; 928.3)$ & 0.43 \\
\hline Epicardiac fat volume $\left(\mathrm{cm}^{3}\right)^{\mathrm{a}}$ & $37.8 \pm 17.2$ & $20.7 \pm 7$ & $<0.001$ \\
\hline L4 vertebral fat volume $\left(\mathrm{cm}^{3}\right)^{\mathrm{a}}$ & $39.19 \pm 9.17$ & $43.2 \pm 18.4$ & 0.52 \\
\hline Epicardial fat on CT $(\mathrm{mm})^{\mathrm{b}}$ & $6(2 ; 15)$ & $6(2 ; 14)$ & 0.49 \\
\hline
\end{tabular}

Notes: ${ }^{a}$ Variables with parametric distribution (mean \pm standard deviation); ${ }^{b}$ Variables with non-parametric distribution (median and interquartile range). Bold values indicate statistical significance.

obtained an intra-class coefficient of correlation (ICC) of 0.89 .

The correlation coefficient between the cardiac ultrasound method and CT scan to assess the epicardiac fat was $\mathrm{r}=0.33, p=0.007$. According to the Bland-Altman test, the mean difference between the two methods was $-0.72 \pm 2.72$. The 95\% upper and lower LOA were 4.2 and $-5.5 \mathrm{~mm}$, respectively (Figure 2).

At 24 weeks after SGLT 2 inhibition, the mean values of the epicardiac fat volume significantly decreased compared

Table 2 Patients Therapies at Baseline

\begin{tabular}{|l|l|l|l|}
\hline $\begin{array}{l}\text { Antidiabetic } \\
\text { Therapy }\end{array}$ & $\begin{array}{l}\text { Number } \\
\text { (Percent) }\end{array}$ & $\begin{array}{l}\text { Other } \\
\text { Therapies }\end{array}$ & $\begin{array}{l}\text { Number } \\
\text { (Percent) }\end{array}$ \\
\hline BG & $50(94.3 \%)$ & $\begin{array}{l}\text { ACE } \\
\text { inhibitors }\end{array}$ & $28(52.8 \%)$ \\
SU & $12(22.6 \%)$ & ARA II & $12(22.6 \%)$ \\
DPP4 inhibitors & $7(13.2 \%)$ & CCB & $11(20.7 \%)$ \\
GLP-I agonists & 0 & STAT & $39(73.5 \%)$ \\
Insulin & $9(16.9 \%)$ & FIB & $10(18.8 \%)$ \\
AG Inhibitors & 0 & PTA & $22(41.5 \%)$ \\
\hline
\end{tabular}

Abbreviations: BG, biguanides; SU, sulfonylureas; DPP4 inhibitors, dipeptidyl peptidase 4 inhibitors; GLP-I agonists, glucagon-like peptid I agonists; AG inhibitors, AlphaGlucosidase Inhibitors; ARA II, Angiotensin II Receptor Antagonists; CCB, calcium channel blockers; STAT, statins; FIB, fibrates; PTA, platelet antiaggregants. to baseline: $37.8 \mathrm{~cm}^{3} \pm 17.2 \mathrm{~cm}^{3}$ vs $20.7 \mathrm{~cm}^{3} \pm 7 \mathrm{~cm}^{3}$, $p<0.001$ (Figure 3). The parameters tested at 6 months are listed in Table 1.

Epicardial fat volume in women at baseline was $35.6 \pm$ $16.7 \mathrm{~cm}^{3}$ vs. $19.7 \pm 5.1 \mathrm{~cm}^{3}$ at 24 weeks follow-up. In men, epi fat volume was $39 \pm 17.5 \mathrm{~cm}^{3}$ before treatment and $21.4 \pm 8.1 \mathrm{~cm}^{3}$ after 6 months of dapagliflozin. The differences between men and women were not statistically significant $(p=0.72)$.

40/53 (75.4\%) patients presented more than 10\% decrease of epicardiac fat volume, $5 / 53(9.5 \%)$ remained stable, while $8 / 53(15.1 \%)$ had more than $10 \%$ increase of epicardiac fat volume. The factors associated with the decrease of the epicardiac fat volume were total cholesterol $(p=0.02)$, LDL c $(p=0.003)$ and the reduction of total weight during the treatment with SGLT 2 inhibitors $(p=0.03)$.

For the type 1 diastolic dysfunction analysis, we excluded the patients with LVEF $<40 \%$ and with significant structural heart disease, so the final analysis included 45 patients (Table 3). 33/45 (73.3\%) had DD1 at baseline, at 6 months 32/45 (71.1\%) had DD1 and after only 1 year of treatment 11/45 (24.4\%) had DD1, $p<0.001$. Patients ( $9 /$ $45,20 \%$ ) who received rescue therapy with association of 


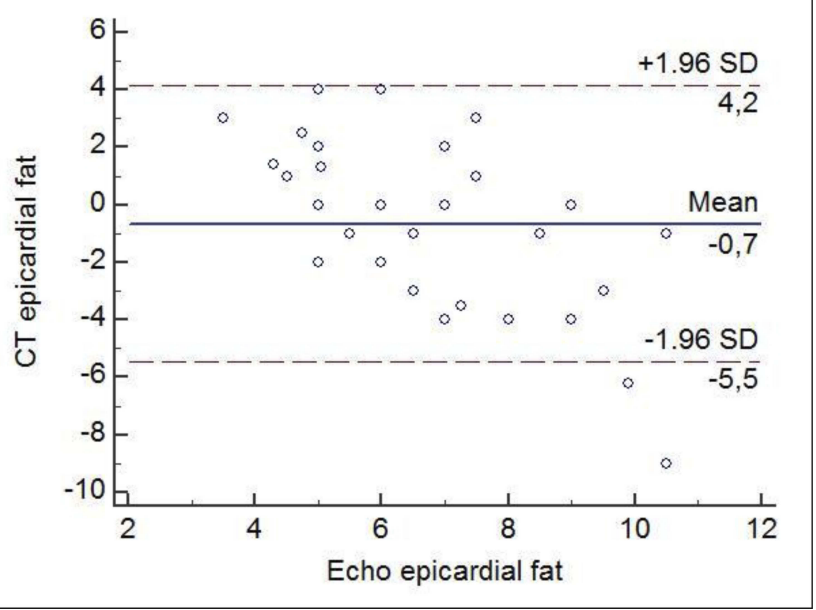

Figure 2 Bland-Altman plot of differences in imagistic assessment of epicardiac fat by two methods: non-contrast CT scan and cardiac ultrasound. The solid line represents the mean of the difference in the epicardiac values. The dashed lines define the LOA. The $95 \%$ upper and lower LOA were 4.2 and -5.5 , respectively.

SGLT 2 inhibitors and DPP 4 inhibitors at the 3- or 6-month follow-up visits had $100 \%$ remission of diastolic dysfunction, compared to the group who received only SGLT 2 inhibitors, $58 \%$ remission $(p=0.04)$. In univariate analysis, epicardiac fat and left atrium volumes were associated with the DD1 remission ( $p=0.02$, respectively $p=0.04)$.

\section{Discussion}

Heart failure is characterized by heterogeneous, nonspecific clinical signs, of various etiologies, which makes its management a challenge both in terms of early

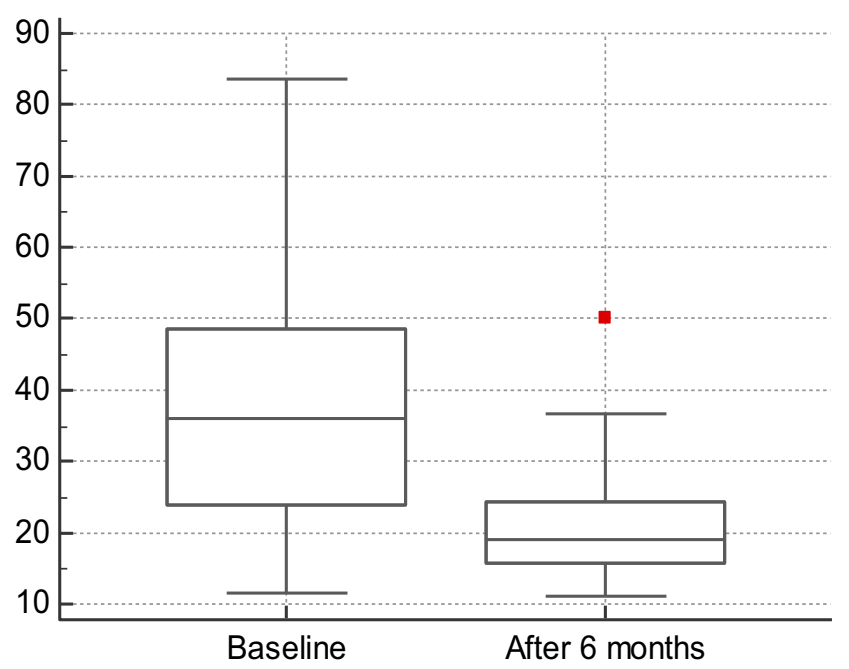

Figure 3 The average differences of epicardiac fat volume at baseline and after 6 months of treatment. On $y$-axis, the scale represents the volume of epicardiac fat, measured in $\mathrm{cm}^{3}$. diagnosis and treatment. Lifestyle, high prevalence of obesity and diabetes in an ageing population led to a higher incidence of heart failure. Of the heart failure phenotypes, left ventricular dysfunction has an increased prevalence of up to $68 \%$ according to the SHORTWAVE study in which 386 patients with T2D, without known cardiovascular disease or signs of ischemia at echo-stress test, were clinically and echocardiographically assessed. ${ }^{16}$ In previous studies, diastolic dysfunction was associated with aging, with an increased duration of diabetes and fasting glycaemia, high blood pressure and increased interventricular septum, with evidence of high obesity, dyslipidemia, and a not very high HbAlc 7.2-7.5\%. ${ }^{17}$ Also, epicardiac fat depots, known as being associated with insulin resistance and coronary artery disease, have proved to induce diastolic dysfunction as a consequence of its local and systemic neurohormonal effects. ${ }^{18}$ From the point of view of the therapeutic management of T2D, SGLT2 inhibitors have proved effective not only in decreasing HbA1c, weight and blood pressure but also in reducing the risk of hospitalization for heart failure, ${ }^{15}$ although the mechanisms by which this molecule acts on the cardiovascular system are not fully understood.

Our study demonstrates that dapagliflozin induced a significant decrease in epicardial tissue volume after 6 months of treatment in both men and women, an effect that was associated with weight loss unconnected to subcutaneous adipose tissue dynamics or abdominal visceral fat. Moreover, in the subject group, the volume of abdominal visceral fat measured at L4 vertebrae, which is an index correlated with cardiovascular risk, ${ }^{19}$ tended to increase among women despite weight loss in general. In women, the reduction of epicardiac fat volume was not associated with the reduction of the waist circumference. There are no clues for this particular lack of association. Recent studies have shown that other gliflozins in this class have led to the reduction of epicardial fat after a short period of treatment, measured by various methods such as bioimpedance (canagliflozin), ${ }^{20}$ nuclear magnetic resonance (luseogliflozin, ipragliflozin), ${ }^{21,22}$ computed tomography (dapagliflozin) $^{24}$ or echocardiography (canagliflozin), ${ }^{20}$ but the mechanisms leading to these results are not fully known. It is assumed that due to the fact that epicardiac fat has a higher turnover of fatty acids, it has a greater sensitivity to the action of SGLT2 inhibitors than visceral tissue in general. $^{23}$ Although nuclear magnetic resonance or computed tomography investigations are highly conclusive, the measurement of thickness of the epicardial fat 
Table 3 The Cardiac Function Evaluated from Baseline to I Year After the Beginning of the Treatment

\begin{tabular}{|l|l|l|l|l|}
\hline Parameter & Baseline & After 6 Months & After I Year & p-Value \\
\hline LA $\left(\mathrm{cm}^{3}\right)$ & $34.93 \pm 12.64$ & $35.01 \pm 6.78$ & $36.24 \pm 11.62$ & 0.61 \\
IVS $(\mathrm{mm})$ & $12.86 \pm 2.16$ & $13.32 \pm 1.36$ & $12.99 \pm 5.57$ & 0.69 \\
TDV $(\mathrm{mL})$ & $115.31 \pm 20.82$ & $114.59 \pm 22.45$ & $109.37 \pm 22.64$ & 0.5 \\
E/A & $0.87 \pm 0.19$ & $0.90 \pm 0.18$ & $1.07 \pm 0.26$ & $<0.001$ \\
Epicardial fat $(\mathrm{mm})$ & $5.95 \pm 1.23$ & $4.25 \pm 1.50$ & $3.01 \pm 1.71$ & $<0.001$ \\
LVEF $(\%)$ & $51.72 \pm 5.60$ & $54.29 \pm 6.43$ & $54.68 \pm 6.93$ & 0.06 \\
RV $(\mathrm{cm})$ & $2.73 \pm 0.15$ & $2.73 \pm 0.17$ & $2.75 \pm 0.25$ & 0.94 \\
\hline
\end{tabular}

Note: Bold values indicate statistical significance.

Abbreviations: LA, left atrium volume; IVS, thickness of interventricular septum; TDV, telediastolic volume; LVEF, left ventricle ejection fraction; RV, right ventricle; E/A, $E$ and $A$ waves ratio.

by echocardiography is more readily available to practitioners because it is a noninvasive, un-expensive, fast method that does not expose the patient to radiation. We observed epicardiac fat thickness in the parasternal, long axis view, subcostal, during one year of SGLT2 inhibition and detected a significant and persistent reduction of epicardiac fat in most of the patients, independent of glycemic control. Most patients had been treated with statins since the beginning of the study; others had been initiated along the way; therefore, weight loss and possible change in the patient's diet had contributed to the improvement in total cholesterol and LDLc, which were associated with a decreased volume of epicardial fat.

In accordance with recent studies, the proportion of diastolic dysfunction in the study group was initially $73 \%$, with the main difference that we particularly screened for type 1 diastolic dysfunction, which represents an impairment of active relaxation of the left ventricle without structural changes in myocardium. It is known that diastolic dysfunction precedes systolic impairment. ${ }^{25,26}$ Our analysis showed a significant improvement of type 1 diastolic dysfunction at 24 weeks, but mostly at one year of treatment with dapagliflozin, from a proportion of $73 \%$ to $24.4 \%$ ( $p<0.001)$, with the observation that patients who received rescue therapy with dapagliflozin and DPP4 inhibitors showed total remission of diastolic dysfunction, whereas the subgroup that remained only on SGLT2 inhibitors showed a remission in only $58 \%$ of the cases. This improvement may be attributed to the reduction of preload and afterload as a result of decreased plasma volume after SGLT2 inhibition. ${ }^{27,28}$ It is believed that the association of dapagliflozin and DPP4 inhibitors is beneficial to the diastolic function ${ }^{29}$ probably because SGLT2 inhibition neutralised certain effects of DPP4 inhibitors on the cardiovascular system. In our study, the volume of epicardial fat and left atrium volume were associated with the remission of diastolic dysfunction type 1. On the same note, Verma et al demonstrated in their study with empagliflozin an important reduction in the mass index of the left ventricle and an improvement in diastolic dysfunction evaluated by e' in a group of type 2 diabetic patients with cardiovascular disease. Another study conducted by Matsunani et al showed that canagliflozin improved diastolic function in a group of patients with $\mathrm{T} 2 \mathrm{D}$ and preserved ejection fraction heart failure, in terms of E/e' and left ventricle mass index, after 12 weeks. In accordance with these studies, Fumitaka Soga et al associated dapagliflozin with a decrease in left atrium volume, left ventricle mass index and with an improvement in diastolic functional parameter $\mathrm{E} / \mathrm{e}^{\prime}$ in a group of patients with heart failure.

The left ventricular ejection fraction showed an improvement trend from an average of $51 \%$ initially to $54 \%$ at the end of the study, but this change did not reach statistical significance.

\section{Study Limitations}

Our single arm study included a relatively small number of patients with heterogeneous characteristics who had been assessed for a relatively short period of time. Patients' recommendations for lifestyle, physical exercise or eating behavior cannot be assumed followed. Also, the metabolic effects of other medications followed by patients cannot be excluded. Therefore, further randomized, clinical cohort studies on larger groups of patients, with a longer evaluation period are needed to validate our findings.

\section{Conclusion}

Our results are demonstrating for the first time the beneficial effect of dapagliflozin on type 1 diastolic dysfunction, in association with a reduction of epicardiac adipose tissue in patients with T2D, independent of glycemic control. The 
results are reinforcing the idea of the beneficial role of SGLT2 inhibitors on cardiac function, which goes beyond the benefits deriving from the glycemic control.

\section{Acknowledgment}

The authors gratefully acknowledge the professionals from the Diabetes Center of the "Pius Brinzeu" Emergency Hospital Timisoara, Romania, for their support in data acquisition.

\section{Disclosure}

The authors report no conflicts of interest in this work.

\section{References}

1. Prati F, Arbustini E, Labellarte A, et al. Eccentric atherosclerotic plaques with positive remodelling have a pericardial distribution: a permissive role of epicardial fat? A three-dimensional intravascular ultrasound study of left anterior descending artery lesions. Eur Heart J. 2003;24(4):329-336.

2. Pezeshkian M, Nouri M, Najjarpour-Jabbari H, et al. Fatty acid composition of epicardial and subcutaneous human adipose tissue. Metab Syndr Relat Disord. 2009;7(2):125-132. doi:10.1089/ met.2008.0056

3. Sacks HS, Fain JN, Holman B, et al. Uncoupling protein-1 and related messenger ribonucleic acids in human epicardial and other adipose tissues: epicardial fat functioning as brown fat. Mol Endocrinol. 2009;23(9):1519-1520. doi:10.1210/mend.23.9.9997

4. Iacobellis G, Corradi D, Sharma AM. Epicardial adipose tissue: anatomic, biomolecular and clinical relationships with the heart Nat Clin Pract Cardiovasc Med. 2005;2(10):536-543. doi:10.1038/ ncpcardio0319

5. Baker AR, Silva NF, Quinn DW, et al. Human epicardial adipose tissue expresses a pathogenic profile of adipocytokines in patients with cardiovascular disease. Cardiovasc Diabetol. 2006;5:1. doi: $10.1186 / 1475-2840-5-1$

6. Mazurek T, Zhang L, Zalewski A, et al. Human epicardial adipose tissue is a source of inflammatory mediators. Circulation. 2003;108 (20):2460-2466. doi:10.1161/01.CIR.0000099542.57313.C5

7. Kremen J, Dolinkova M, Krajickova J, et al. Increased subcutaneous and epicardial adipose tissue production of proinflammatory cytokines in cardiac surgery patients: possible role in postoperative insulin resistance. J Clin Endocrinol Metab. 2006;91(11):4620-4627. doi:10.1210/jc.2006-1044

8. Chaldakov GN, Fiore M, Stankulov IS, et al. Neurotrophin presence in human coronary atherosclerosis and metabolic syndrome: a role for NGF and BDNF in cardiovascular disease? Prog Brain Res. 2004;146:279-289.

9. Gruzdeva O, Uchasova E, Dyleva Y, et al. Relationships between epicardial adipose tissue thickness and adipo-fibrokine indicator profiles post-myocardial infarction. Cardiovasc Diabetol. 2018;17(1):40 doi:10.1186/s12933-018-0679-y

10. Xu L, Xu Y, Coulden R, et al. Comparison of epicardial adipose tissue radiodensity threshold between contrast and non-contrast enhanced computed tomography scans: a cohort study of derivation and validation. Atherosclerosis. 2018;275:74-79. doi:10.1016/j. atherosclerosis.2018.05.013

11. Rosito GA, Massaro JM, Hoffmann U, et al. Pericardial fat, visceral abdominal fat, cardiovascular disease risk factors, and vascular calcification in a community-based sample: the Framingham heart study. Circulation. 2008;117(5):605-613. doi:10.1161/CIRCULATIONAHA. 107.743062
12. Mookadam F, Goel R, Alharthi MS, Jiamsripong P, Cha S. Epicardial fat and its association with cardiovascular risk: a cross-sectional observational study. Heart Views. 2010;11(3):103-108. doi:10.4103/ 1995-705X.76801

13. Matsushita K, Blecker S, Pazin-Filho A, et al. The association of hemoglobin a1c with incident heart failure among people without diabetes: the atherosclerosis risk in communities study. Diabetes. 2010;59(8):2020-2026. doi:10.2337/db10-0165

14. Seferovic PM, Petrie MC, Filippatos GS, et al. Type 2 diabetes mellitus and heart failure: a position statement from the heart failure association of the European Society of Cardiology. Eur J Heart Fail. 2018;20(5):853-872. doi:10.1002/ejhf.2018.20.issue-5

15. Zelniker TA, Wiviott SD, Raz I, et al. SGLT2 inhibitors for primary and secondary prevention of cardiovascular and renal outcomes in type 2 diabetes: a systematic review and meta-analysis of cardiovascular outcome trials. Lancet. 2019;393(10166):31-39. doi:10.1016/ S0140-6736(18)32590-X

16. Faden G, Faganello G, De Feo S, et al. The increasing detection of asymptomatic left ventricular dysfunction in patients with type 2 diabetes mellitus without overt cardiac disease: data from the SHORTWAVE study. Diabetes Res Clin Pract. 2013;101 (3):309-316. doi:10.1016/j.diabres.2013.07.004

17. Celentano A, Vaccaro O, Tammaro P, et al. Early abnormalities of cardiac function in non-insulin-dependent diabetes mellitus and impaired glucose tolerance. Am J Cardiol. 1995;76(16):1173-1176. doi:10.1016/S0002-9149(99)80330-0

18. Ferrara D, Montecucco F, Dallegri F, Carbone F. Impact of different ectopic fat depots on cardiovascular and metabolic diseases. J Cell Physiol. 2019. doi:10.1002/jep.28821

19. Gao Y, Wang Y-C, Lu C-Q, Zeng C, Chang D, Ju S. Correlations between the abdominal fat-related parameters and severity of coronary artery disease assessed by computed tomography. Quant Imaging Med Surg. 2018;8(6):579-587. doi:10.21037/qims

20. Yagi $S$, Hirata $Y$, Ise $T$, et al. Canagliflozin reduces epicardial fat in patients with type 2 diabetes mellitus. Diabetol Metab Syndr. 2017;9:78. doi:10.1186/s13098-017-0275-4

21. Bouchi R, Terashima M, Sasahara Y, et al. Luseogliflozin reduces epicardial fat accumulation in patients with type 2 diabetes: a pilot study. Cardiovasc Diabetol. 2017;16(1):32. doi:10.1186/s12933-017-0516-8

22. Fukuda $T$, Bouchi R, Terashima $M$, et al. Ipragliflozin reduces epicardial fat accumulation in non-obese type 2 diabetic patients with visceral obesity: a pilot study. Diabetes Ther. 2017;8(4):851-861. doi:10.1007/s13300-017-0279-y

23. Marchington JM, Pond CM. Site-specific properties of pericardial and epicardial adipose tissue: the effects of insulin and high-fat feeding on lipogenesis and the incorporation of fatty acids in vitro. Int J Obes. 1990;14(12):1013-1022.

24. Sato T, Aizawa Y, Yuasa S, et al. The effect of dapagliflozin treatment on epicardial adipose tissue volume. Cardiovasc Diabetol. 2018;17 (1):6. doi:10.1186/s12933-017-0658-8

25. Suresh G, Alva R, Prakash P, Saya R. Prevalence of asymptomatic left ventricular diastolic dysfunction in type 2 diabetic patients and healthy controls: a comparative study. Arch Med Health Sci. 2017;5(1):30.

26. Francis GS. Diabetic cardiomyopathy: fact or fiction? Heart. 2001;85 (3):247-248. doi:10.1136/heart.85.3.247

27. Pham SV, Chilton RJ. EMPA-REG OUTCOME: the cardiologist's point of view. Am J Cardiol. 2017;120(1s):S53-s58. doi:10.1016/j. amjcard.2017.05.011

28. Rajasekeran H, Lytvyn Y, Cherney DZ. Sodium-glucose cotransporter 2 inhibition and cardiovascular risk reduction in patients with type 2 diabetes: the emerging role of natriuresis. Kidney Int. 2016;89 (3):524-526. doi:10.1016/j.kint.2015.12.038

29. Matsutani D, Sakamoto M, Kayama Y, Takeda N, Horiuchi R, Utsunomiya K. Effect of canagliflozin on left ventricular diastolic function in patients with type 2 diabetes. Cardiovasc Diabetol. 2018;17(1):73. doi:10.1186/s12933-018-0717-9 


\section{Publish your work in this journal}

Diabetes, Metabolic Syndrome and Obesity: Targets and Therapy is an international, peer-reviewed open-access journal committed to the rapid publication of the latest laboratory and clinical findings in the fields of diabetes, metabolic syndrome and obesity research. Original research, review, case reports, hypothesis formation, expert opinion and commentaries are all considered for publication. The manuscript management system is completely online and includes a very quick and fair peer-review system, which is all easy to use. Visit http://www.dovepress.com/testimonials.php to read real quotes from published authors. 